\title{
Playing the waiting game
}

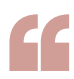

The overall goal of our approach is to establish culture conditions that maintain somatic stem cell potency

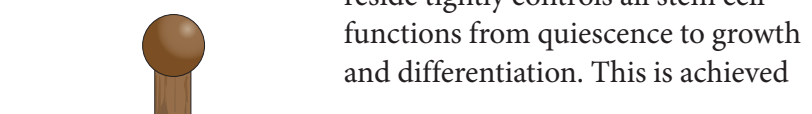

through the complex interplay of mechanical and biochemical factors. Despite the therapeutic importance of promoting and maintaining quiescence for improved potency, most attempts to bioengineer artificial niches have focused on directing cell fate. By contrast, "The overall goal of our approach is to establish culture conditions that maintain somatic stem cell potency," explains Rando.

The approach involves the identification and recapitulation of the biochemical and mechanical conditions required for quiescence of mouse muscle stem cells (MuSCs). The researchers first analysed and established molecular signatures (that is, the genes that are highly expressed) of both quiescent and activated MuSCs. They then screened 50 proteins that are either known or thought to promote quiescence, and shortlisted 10 on the basis of their ability to prevent proliferation. Through combinatorial screening, they identified an optimal composition (a 'quiescence medium') in which the MuSCs were molecularly very similar to freshly isolated quiescent MuSCs.

Having established the optimal chemical composition, the researchers determined the substrate elasticity that best preserved quiescence.
Using a microfluidic method, they then fabricated artificial collagen I fibres with the desired elasticity that mimic myofibres in the native MuSC niche. Stem cells cultured on the fibres in the quiescence medium not only maintained quiescence for a longer duration in culture, but they also exhibited substantially enhanced potency in terms of engraftment, self-renewal and tissue regeneration after transplantation in mice (compared with native-fibre-associated MuSCs and freshly isolated MuSCs). "We were able to engineer conditions that markedly enhance the potency of muscle stem cells so that, upon transplantation, they were much more effective in generating new muscle cells after an injury to the muscle tissue," comments Rando. Importantly, they were able to demonstrate that the approach developed for mouse MuSCs also worked for human MuSCs.

The next phase of this research will involve up-scaling and process optimization: "Our plan is to continue to develop these methodologies to create as simple a system as possible to allow these culture conditions to be easily scaled from use in mice to use in larger animals and humans, with the ultimate goal of facilitating effective stem cell therapies for the treatment of injuries and degenerative diseases in humans." Moreover, this approach is expected to be effective not only for MuSCs, but for all somatic stem cells.

\section{Adam Brotchie}

ORIGINAL ARTICLE(S) Quarta, M. et al. An artificial niche preserves the quiescence of muscle stem cells and enhances their therapeutic efficacy. Nat. Biotechnol. http://dx.doi.org/10.1038/nbt.3576 (2016) 